\title{
Association between LRRK2 and 4E-BP1 protein levels in normal and malignant cells
}

\author{
BERTA PONS $^{1,2^{*}}$, GEMMA ARMENGOL $^{3 *}$, MARK LIVINGSTONE $^{4}$, \\ LAURA LÓPEZ ${ }^{1,2}$, LAURA COCH $^{1}$, NAHUM SONENBERG ${ }^{4 * *}$ and SANTIAGO RAMÓN Y CAJAL ${ }^{1 * *}$ \\ ${ }^{1}$ Department of Pathology, Vall d'Hebron University Hospital, ${ }^{2}$ Department of Biochemistry and Molecular \\ Biology, Faculty of Biosciences, Universitat Autónoma de Barcelona, ${ }^{3}$ Department of Animal Biology, \\ Plant Biology and Ecology, Universitat Autònoma de Barcelona, Barcelona, Spain; ${ }^{4}$ Department of \\ Biochemistry and McGill Cancer Centre, McGill University, Montreal, QC H3A 1A3, Canada
}

Received June 6, 2011; Accepted July 27, 2011

DOI: 10.3892/or.2011.1462

\begin{abstract}
Translational control is a crucial component of cancer development and progression. Eukaryotic initiation factor (eIF) 4E mediates eIF4F association with the mRNA 5' cap structure to stimulate cap-dependent translation initiation. The eIF4E-binding protein, 4E-BP1, regulates cap-dependent translation through its phosphorylation at multiple sites. It has been described that some human carcinomas present a high level of p-4E-BP1, not always associated with high levels of p-mTOR. These previous observations suggest that other kinases could be involved in 4E-BP1 phosporylation. Investigation in new kinases that could be implicated in $4 \mathrm{E}-\mathrm{BP} 1$ phosphorylation and mechanisms that affect $4 \mathrm{E}-\mathrm{BP} 1$ stability is important to understand the role of eIF4E in cell transformation. In this study, we examined 48 kinases that could be involved in 4E-BP1 phosphorylation and stability. The screening study was based on analysis of 4E-BP1 status after inhibition of these kinases in a breast carcinoma cell line. Several kinases affecting 4E-BP1 stability (LRRK2, RAF-1, p38 $\gamma$, GSK3 $\beta$, AMPK $\alpha$, PRKACA and PRKACB) and 4E-BP1 phosphorylation (CDK1, PDK1, SRC, PRKCB1, PAK2, p38 $\beta$, PRKCA and CaMKKB) were identified. These findings provide evidence that $4 \mathrm{E}-\mathrm{BP} 1$ can be regulated and stabilized by multiple kinases implicated in several cell signaling pathways. We focus on the finding that LRRK2 down-regulation was associated with a clearly decreased 4E-BP1 protein (and
\end{abstract}

Correspondence to: Dr Santiago Ramon y Cajal, Department of Pathology, Vall d'Hebron University Hospital, Pg. Vall d'Hebron 119-129, 08035-Barcelona, Spain

E-mail: sramon@vhebron.net

*Contributed equally

*** Shared senior authorship

Key words: 4E-BP1, kinases, LRRK2, protein degradation, proteasome, MG132 not with mRNA down-regulation). Importantly, knockdown of LRRK2 associated with high proliferative rate in normal cells and treatment with rapamycin and/or proteosome inhibition suppressed 4E-BP1 protein degradation. These results offer new insights into the regulation of total and phosphorylated 4E-BP1.

\section{Introduction}

Aberrant translation is emerging as an important process related to cancer progression. One of the most highly regulated steps in protein translation is at the initiation level, and a main factor is the control of eukaryotic initiation factor complex (eIF4F) formation. eIF4F is comprised of a large scaffolding protein, eIF4G, an mRNA helicase, eIF4A, and the protein eIF4E, which mediates binding of eIF4F to the 5' cap structure of mRNAs. eIF4F formation is regulated by three binding proteins, the 4E-BPs, including 4E-BP1, 4E-BP2 and 4E-BP3, with 4E-BP1 being the predominant form. The 4E-BPs compete with eIF4G for eIF4E and block eIF4F formation. Binding of 4E-BP1 to eIF4E is regulated by phosphorylation. 4E-BP1 phosphorylation results in eIF4E release and activation of cell protein synthesis.

There is considerable interest in the 4E-BPs and their relation to cancer, stimulated by a range of recent findings. Overexpression of $4 \mathrm{E}-\mathrm{BP} 1$ reverses the transformed phenotype of cells overexpressing eIF4E, thus, indicating that $4 \mathrm{E}-\mathrm{BP} 1$ acts as a tumor suppressor (1). Moreover, some studies have shown that 4E-BP1, together with S6K, regulates animal growth and cell size (2), and that 4E-BP1 appears to be important in cell survival $(3,4)$. In previous studies in large series of breast, ovarian, prostate, and endometrial tumors, we observed that 4E-BP1 and phospho-4E-BP1 (p-4E-BP1) expression was associated with malignant progression and an adverse prognosis, regardless of the upstream oncogenic alterations (5-8). Other authors have reported similar results in breast carcinomas, melanomas, and prostate cancer (9-12). For example, in a study on breast cancer, phosphorylation of AKT, mTOR, and 4E-BP1 was associated with tumor development and progression (11). Kremer et al (12) investigated the expres- 
sion patterns of several biomarkers of the mTOR pathway in prostate cancer and observed that 4E-BP1 levels, in combination with mTOR and PTEN activation, were one of the best biomarkers of prostate intraepithelial neoplasia.

The importance of 4E-BP1 lies in its control of cap-dependent translation initiation complex assembly. It is important to point out that in humans, 4E-BP1 has six phosphorylation sites, including Thr 37, Thr 46, Ser 65, Thr 70, Ser 83, and Ser $112(13,14)$. It is likely that mTOR is the main phosphorylation pathway of 4E-BP1 (15), although other kinases may be implicated, such as cyclin-dependent kinase $1(16,17)$, ataxia-telangiectasia mutated (ATM) (18), PI3K-AKT $(19,20)$, ERK1/2 (21), and perhaps other, still unidentified, kinases. Imai et al (22) have reported that 4E-BP1 may be a substrate of LRRK2, a protein that is mutated in Parkinson disease. Moreover, oxidative stress and other stimuli that have an impact on protein translation affect 4E-BP phosphorylation. Sequential phosphorylation of 4E-BP1 (Thr 37/46>Thr 70 $>$ Ser 65$)$ has also been proposed $(23,24)$. Although the regulatory mechanisms involved in 4E-BP1 phosphorylation are not fully understood, it may be that a combination of phosphorylation events is required to dissociate 4E-BP1 from eIF4E (24). There is evidence suggesting that 4E-BP1 phosphorylation on Thr 70 and Ser 65 is required to release eIF4E from 4E-BP1 (25). Although these findings imply that 4E-BP1 deregulation may have a causal effect on tumorigenesis, it remains unresolved whether these changes directly contribute to cancer formation. In this regard, previous studies in human carcinomas did not observe a clear correlation between p-4E-BP1 and phosphorylation of mTOR or MAPK (mitogen-activated protein) (5). This suggests that other kinases and biochemical pathways could be involved in 4E-BP1 phosphorylation in tumors. Given that eIF4E availability is controlled by the total amount of 4E-BP1 and by 4E-BP1 phosphorylation, the study of kinases that could be implicated in the phosphorylation and stability of this protein could help to elucidate the contribution of eIF4E in cell transformation and human cancer.

In this study, the effects of inhibiting 48 cellular kinases on 4E-BP1 phosphorylation and stability were investigated. The findings indicate that multiple kinases implicated in various cell signaling pathways can participate in these outcomes: CDK1, PDK1, SRC, PRKCB1, PAK2, p38 $\beta$, PRKCA and CaMKKB (affecting 4E-BP1 phosphorylation) and LRRK2, RAF-1, p38 $\gamma$, GSK3 $\beta$, AMPK $\alpha$, PRKACA and PRKACB (affecting total 4E-BP1 stability). In addition, LRRK2 was seen to exert a regulating effect on protein translation, stabilizing total 4E-BP1 in vitro. Moreover, the stabilization events appear to be functionally important for growth control in normal cells.

\section{Materials and methods}

Cell lines and cell culture. The following cell lines were used in the study: TIG3 human fibroblasts (kindly donated by Dr D. Peeper, The Netherlands Cancer Institute, Amsterdam), 769-P kidney carcinoma cells (ATCC, American Type Culture Collection, Middlesex, UK), MDA-MB-231 breast carcinoma cells (ATCC) and DLD-1 colon carcinoma cells (ATCC). Cells were maintained in standard DMEM (Dulbecco's modified Eagle's medium) growth medium supplemented with $10 \%$ fetal calf serum and antibiotics.
siRNA transfection. siRNAs for 48 kinases used to identify new candidates for phosphorylation or stabilization of 4E-BP1 were purchased from Qiagen (Valencia, CA, USA). All siRNAs were validated, with the exception of LRRK2, for which two different siRNAs were used. In addition to the siRNA for each kinase, a siRNA non-target (NT) (1022076, Qiagen) was used as a negative control and mTOR siRNA and 4E-BP1 siRNA were used as positive controls. MDA-MB-231 cells $\left(18 \times 10^{4}\right)$ were seeded in a 6-well plate and $30 \mathrm{nM}$ of each siRNA were transfected using Lipofectamine 2000 Reagent (Invitrogen, Paisley, UK), following the manufacturer's instructions. Each kinase was inhibited by one or two siRNA, and 4E-BP1 status was analyzed by Western blot analysis $72 \mathrm{~h}$ later. Each kinase inhibition was performed 3 times with similar results regarding the effect on $4 \mathrm{E}-\mathrm{BP} 1$ phosphorylation and $4 \mathrm{E}-\mathrm{BP} 1$ stabilization.

To study the effect of LRRK2 in more detail and to validate the results of the screening, LRRK2 was inhibited with the siRNA 1 used in the screening (SI00624526, Qiagen) and with a new siRNA (siRNA 3) (SI00624540, Qiagen) that had not been used in the first screening.

shLRRK2-pLKO.1 and shNT-pLKO.1 construction. The pLKO.1-shLRRK2_1 and pLKO.1-shLRRK2_3 constructions were generated using the sequences of the two different LRRK2 siRNAs, LRRK2 siRNA 1 sequence: 5'gatcc GCTCGTCGACTTATACGTGTAATTCAAGAGATTACAC GTATAAGTCGACGAGTTTTTTACGCGTg3', and LRRK2 siRNA 3 sequence: 5'gatccGCAGAATTTCATCATAAGC TAATTCAAGAGATTAGCTTATGATGAAATTCTGT TTTTTACGCGTg3' (both from Qiagen). The shRNA for LRRK2 1 and LRRK2 3 were designed and cloned into the pLKO.1 puro vector (Sigma-Aldrich, Taufkirchen, Germany). A pLKO.1-sh non-target (NT) vector (Sigma-Aldrich) was used as control for lentivirus infection.

Lentivirus-based shRNA transduction. To investigate whether LRRK2 inhibition could affect cell proliferation, lentivirusbased transduction on TIG3 cells was carried out using a packaging cell line, HEK293T, by cotransfection of pCMVdR8.91 dvpr and VSV-G (Clontech, Saint-Germain-en-Laye, France). After performing two consecutive virus infections, cells were selected with puromycin for 3 days.

Retroviral plasmid construct. pBABE-4E-BP1 (4 Ala) contains a mutated form of 4E-BP1. The mutations are on the four most relevant 4E-BP1 phosphorylation sites: Thr 37/46, Thr 70, and Ser 65. The pBABE-4E-BP1 (4Ala) contains two amino terminal hemagglutinin (HA) tags.

Retroviral transduction. To generate an MDA-MB-231 cell line that stably expressed a mutant 4E-BP1 unable to phosphorylate, the pBABE-4E-BP1 (4 Ala) and pBABE constructs were transiently transfected into a packaging cell line (GP-293, Clontech, Heidelberg, Germany) according to the manufacturer's instructions. For retroviral infection, cell monolayers were incubated in the presence of the retrovirus-containing supernatant and $4 \mu \mathrm{g} / \mathrm{ml}$ polybrene (Sigma-Aldrich) for $24 \mathrm{~h}$. Infection was repeated the next day. Twenty-four hours after the second infection, medium supplemented with puromycin 
(0.7 $\mu \mathrm{g} / \mathrm{ml}$, Sigma Aldrich) was added, and cells underwent selection for 3 days to eliminate uninfected cells. After the transduction, MDA-MB-231 cells overexpressing pBABE4E-BP1 (4 Ala) were transfected with LRRK2 siRNAs or NT siRNA and the 4E-BP1 status was analyzed by Western blot analysis $72 \mathrm{~h}$ later.

Growth curves. Two days after selection, TIG3 cells were counted, and then seeded $\left(1 \times 10^{5}\right.$ cells $)$ in duplicate every 3 days. Population doubling (PD) was determined by the following formula: $P D=\log \left(\mathrm{N}_{\mathrm{f}} / \mathrm{N}_{\mathrm{i}}\right) / \log 2$, where $\mathrm{N}_{\mathrm{f}}$ is the number of cells counted and $\mathrm{N}_{\mathrm{i}}$ is the number of cells seeded. Cumulative population doubling level (PDL) numbers represent the sum of PDs from previous passages. Each curve was performed at least twice with similar results, and each time point was determined in duplicate.

Cell treatments. MDA-MB-231 cells were transfected with LRRK2 siRNAs or NT siRNA, and $48 \mathrm{~h}$ after transfection, $18 \times 10^{4}$ cells were seeded in a 6 -well plate. The next day, cells were treated with MG132 $30 \mu \mathrm{M}$ (\#1748, Tocris Bioscience, Ellisville, MO) or rapamycin $30 \mathrm{nM}$ (Cell Signaling, Beverly, MA) for $6 \mathrm{~h}$. Control cells received an equal volume of dimethyl sulfoxide or water. To inhibit cellular phosphatases, $18 \times 10^{4}$ MDA-MB-231 cells were seeded in a 6-well plate and incubated for $24 \mathrm{~h}$ with $5 \mathrm{mM} \mathrm{NaF}$. Control cells received an equal volume of water.

Western blot analysis. Lysates were obtained from the cells. Subconfluent cells were lysed in lysis buffer (HEPES 50 mM, pH 7.5; NaCl 150 mM; 1\% Triton X; EDTA 1 mM; $10 \%$ glycerol) in the presence of protease and phosphatase inhibitors. After clearing the lysates by centrifugation, protein concentrations were determined using the Bradford assay (Bio-Rad Protein Assay, Munich, Germany). Protein (30 $\mu \mathrm{g})$ was denatured and resolved on sodium dodecyl sulfate (SDS) polyacrylamide gel electrophoresis (PAGE) and transferred to polyvinylidene fluoride membranes (PVDF) (Bio-Rad). Western blot analysis was then performed following standard procedures. The primary antibodies used were anti-4E-BP1 antibody (\#9452, Cell Signaling, diluted 1:1000), anti-p4E-BP1 Ser 65 (\#9451, Cell Signaling, diluted 1:1000), anti p-4E-BP1 Thr 37/46 (\#9459, Cell Signaling, diluted 1:1000), anti-4E-BP2 (\#2845, Cell Signaling, diluted 1:100), and antiLRRK2 (AB9682, Millipore, Madrid, Spain, diluted 1:5000). Anti-actin (CP01, Calbiochem, Darmstadt, Germany; diluted 1:7000) was used as the loading control. The secondary antibodies used were donkey anti-rabbit IgG-HRP (NA9340, Amersham Pharma-Biotech, Uppsala, Sweden; diluted 1:2000) and donkey anti-mouse IgG-HRP (NA9340, Amersham Pharma-Biotech; diluted 1:2000). Bound antibodies were visualized with an enhanced chemiluminescence detection kit (Amersham Pharma-Biotech).

$R N A$ extraction and quantitative $R T-P C R$. To investigate transcriptional regulation of 4E-BP1 after LRRK2 inhibition in MDA-MB-231 cells, total RNA was isolated from cells with the RNeasy Mini Kit (Qiagen) following the manufacturer's instructions. Random primers and SuperScript II reverse transcriptase (Invitrogen) were used to carry out cDNA synthesis from $1.5 \mu \mathrm{g}$ of total RNA. 4E-BP1 and LRRK2 expression was detected using Taqman Gene Expression Assay (Hs00607050_m1, Hs00411197_m1, Applied Biosystems, Carlsbad, CA, USA, respectively). An ABI PRISM 7000 instrument (Applied Biosystems) was used for relative quantification analysis, and the data were analyzed with the 7000 Sequence Detection Software, v.1.2.3 (Applied Biosystems). The PCR cycling program consisted of denaturing at $95^{\circ} \mathrm{C}$ for $10 \mathrm{~min}$ and 40 cycles at $95^{\circ} \mathrm{C}$ for $15 \mathrm{sec}$, and annealing and elongation at $60^{\circ} \mathrm{C}$ for $1 \mathrm{~min}$. The reactions were done in triplicate. Previously, a Taqman Human Endogenous Control Plate (Applied Biosystems) was performed to determine which endogenous controls showed less variation between samples. POLR2A (Hs00172187_m1; Applied Biosystems) was the endogenous control chosen. Target and reference genes showed similar, nearly $100 \%$ amplification efficiencies (data not shown). Therefore, the $\Delta \Delta \mathrm{CT}$ method was considered appropriate for relative gene expression analysis.

\section{Results}

Primary screening to identify candidate kinases for $4 E-B P 1$ phosphorylation or stabilization. Forty-eight kinases were screened using MDA-MB-231 cells to identify new candidates for phosphorylation or stabilization of $4 \mathrm{E}-\mathrm{BP} 1$. The validated siRNAs included kinases from the CAMK group (calcium/calmodulin-dependent protein kinases), AGC group (containing PKA, PKG, and PKC families), CMGC group (containing CDK, MAPK, GSK3, and CLK families), TKL group (tyrosine kinase-like), TK group (tyrosine kinases), STE group (homologs of yeast sterile 7, sterile 11, and sterile 20 kinases), atypical group (kinases with no sequence similarity with the other kinase groups), and other kinases (those that do not belong to a specific group, such as IKK and TBK1).

For each Western blot analysis, rapamycin treatment and mTOR siRNA controls were confirmed to induce 4E-BP1 dephosphorylation, and 4E-BP1 siRNA was used to confirm down-regulation of 4E-BP1 and specificity of the antibody. Following kinase inhibition, an effect on 4E-BP1 that was not reproducible at least twice was not considered positive. Kinases that seemed to directly or indirectly phosphorylate 4E-BP1 were CDK1, PDK1, SRC, PRKCB1, PAK2, p38 $\beta$, PRKCA, and CaMKKB (data not shown). Kinases that seemed to affect 4E-BP1 stability were LRRK2, RAF-1, p38 $\gamma$, GSK3 $\beta$, AMPK $\alpha$, PRKACA and PRKACB (data not shown).

LRRK2 inhibition validation. After this first screening, we decided to study the potential effect of LRRK2 on 4E-BP1 stability in more detail. Because the LRRK2 siRNAs used in the screening had not been previously validated, we performed a Western blot analysis against LRRK2 (Fig. 1). The results showed that LRRK2 was totally inhibited after transfection and the total amount of 4E-BP1 was down-regulated.

LRRK2 inhibition causes 4E-BP1 down-regulation in different cell types. After validating the results obtained in the screening with LRRK2, we investigated whether LRRK2 inhibition could affect 4E-BP1 stability in different cell types. First, we inhibited LRRK2 in MDA-MB-231 cells with the siRNA 1 used in the screening and with a new siRNA 


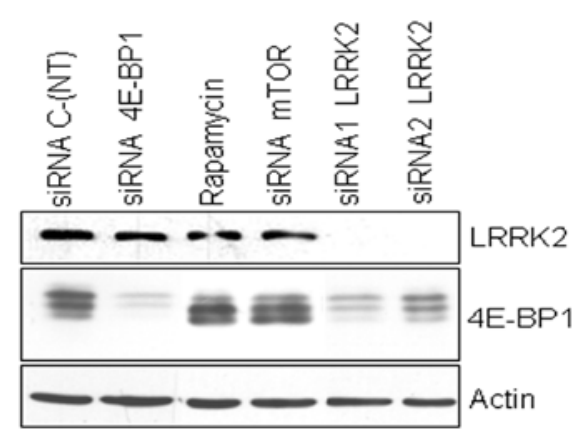

Figure 1. 4E-BP1 status after LRRK2 inhibition in MDA-MB-231, a breast carcinoma cell line. 4E-BP1 was down-regulated after LRRK2 inhibition with siRNA 1 and siRNA 2 used in the first screening. LRRK2 knockdown by siRNA was checked by Western blot analysis.

(siRNA 3). We observed that LRRK2 inhibition with the new siRNA had the same effect as was observed previously in the screening with siRNA 1 and siRNA 2 (Fig. 2A). Therefore, these results reconfirmed the effect of LRRK2 on the total amount of 4E-BP1 in MDA-MB-231 cells.

We then investigated whether LRRK2 inhibition affected 4E-BP1 stability in other cell types. The same outcome on 4E-BP1 stability was produced when LRRK2 was inhibited in 769-P, TIG3 and DLD-1 cells (Fig. 2). Interestingly, in the DLD1 cell line, the down-regulation of 4E-BP1 was lower than in others cell lines and a weak hyperphosphorylated band could be observed after $72 \mathrm{~h}$ of siRNA 3 LRRK 2 transfection (Fig. 2C). Each LRRK2 inhibition in the different cell types tested was performed 3 times with similar results regarding the effect on $4 \mathrm{E}-\mathrm{BP} 1$.

LRRK2 inhibition induces cell proliferation. Because LRRK2 inhibition affected 4E-BP1 stability in more than one cell type, we then investigated whether LRRK2 inhibition could affect cell proliferation. To this end, normal TIG3 cells were infected with pLKO.1-shLRRK2_1, pLKO.1-shLRRK2_3 or pLKO.1shNT, and LRRK2 inhibition was checked by Western blot analysis. TIG3 cells with inhibited LRRK2 proliferated faster than control TIG3 cells (Fig. 3). These results were confirmed with the two shLRRK2 lentivirus vectors. Western blot analysis was also used to determine the effect of LRRK2 inhibition on 4E-BP1 stability. The total amount of 4E-BP1 protein was found to dramatically decrease, whereas surprisingly, the total amount of 4E-BP2 was not affected.

Mode of action of LRRK2. As shown in Fig. 3, LRRK2 inhibition affected 4E-BP1 stability in a very specific manner. We then set out to determine what mechanism could be implicated in $4 \mathrm{E}-\mathrm{BP} 1$ degradation. First, we investigated if the total amount of mRNA of 4E-BP1 was affected after LRRK2 inhibition in MDA-MB-231 cells (Fig. 4). Quantitative RT-PCR analysis showed that LRRK2 was down-regulated after its inhibition with the two siRNAs tested. Interestingly, 4E-BP1 was not down-regulated. These results indicated that LRRK2 inhibition did not affect the total amount of 4E-BP1 mRNA. We then studied post-transcriptional 4E-BP1 regulation after LRRK2 inhibition. Previous studies suggested that phosphorylated forms of 4E-BP1 are more susceptible
A

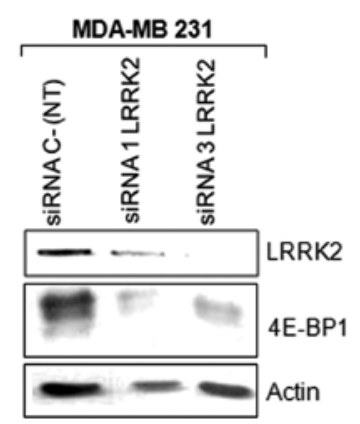

B

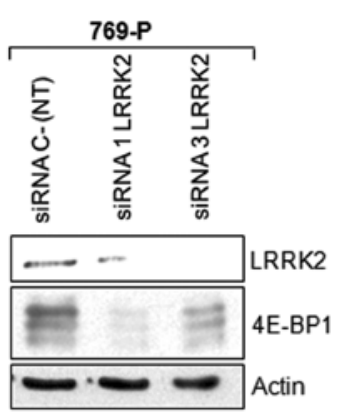

C

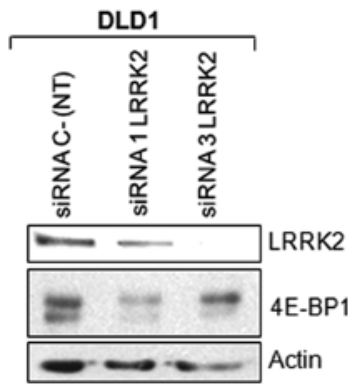

D

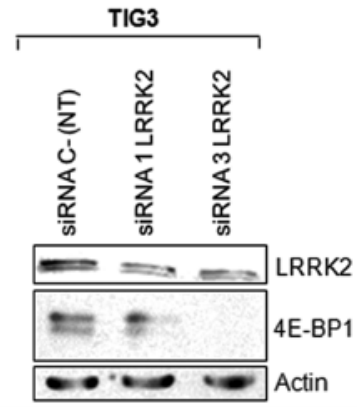

Figure 2. Effect of LRRK2 inhibition on different cell types. (A), 4E-BP1 status after LRRK2 inhibition in MDA-MB-231. 4E-BP1 was down-regulated after LRRK2 inhibition with siRNA 1 (same siRNA used in screening) and with another siRNA (siRNA 3) not used in the first screening. (B), 4E-BP1 status after LRRK2 inhibition in kidney carcinoma cells (769-P). 4E-BP1 was down-regulated in 769-P cells after LRRK2 inhibition. (C), 4E-BP1 status after LRRK2 inhibition in colon carcinoma cells (DLD1). 4E-BP1 was down-regulated in DLD1 cells after LRRK2 inhibition. (D), 4E-BP1 status after LRRK2 inhibition in human fibroblast cells (TIG3). 4E-BP1 was downregulated after LRRK2 inhibition.

to degradation via the proteasome (26-29). To test whether 4E-BP1 degradation occurred via the proteasome, MDA-MB 231 cells with transiently inhibited LRRK2 were treated with MG132, a proteasome inhibitor. In addition, to determine whether hypophosphorylation of 4E-BP1 could inhibit its degradation, cells were treated with rapamycin. As it is shown in Fig. 5A, both treatments restored the total protein levels of 4E-BP1 in MDA-MB-231 cells when LRRK2 was downregulated. This suggested that $4 \mathrm{E}-\mathrm{BP} 1$ degradation following LRRK2 inhibition occurred via the proteasome. The fact that rapamycin treatment had an inhibitory effect on 4E-BP1 loss could indicate that disappearance of 4E-BP1 following LRRK2 inhibition is likely a direct consequence of increased phosphorylation. To address this issue, cells were incubated for $24 \mathrm{~h}$ with $5 \mathrm{mM} \mathrm{NaF}$, a phosphatase inhibitor (Fig. 5B). This treatment caused total 4E-BP1 protein degradation (the same effect was observed on total 4E-BP1 after LRRK2 inhibition). Moreover, overexpression of mutant 4E-BP1 unable to phosphorylate (pBABE-4E-BP1 4 Ala) was not degraded when LRRK2 was down-regulated. There were no differences in the exogenous levels of 4E-BP1 mutant when the LRRK2 was inhibited (see the HA-4E-BP1 in the Fig. 5C).

\section{Discussion}

The activity of 4E-BP1 in controlling protein translation is regulated mainly by its phosphorylation and probably by the 


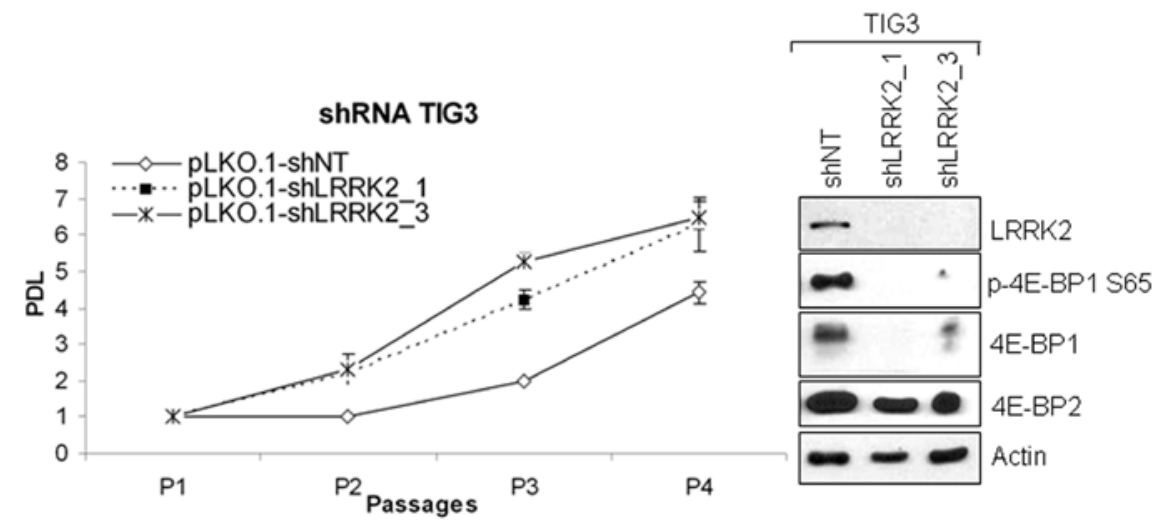

Figure 3. Effect of LRRK2 inhibition on cell proliferation. TIG3 proliferation was significantly increased when LRRK2 was inhibited. These results were confirmed with two shLRRK2 lentiviral vectors. Western blot analysis shows LRRK2 down-regulation and its effect on 4E-BP1 stability. The status of 4E-BP2 was also analyzed.

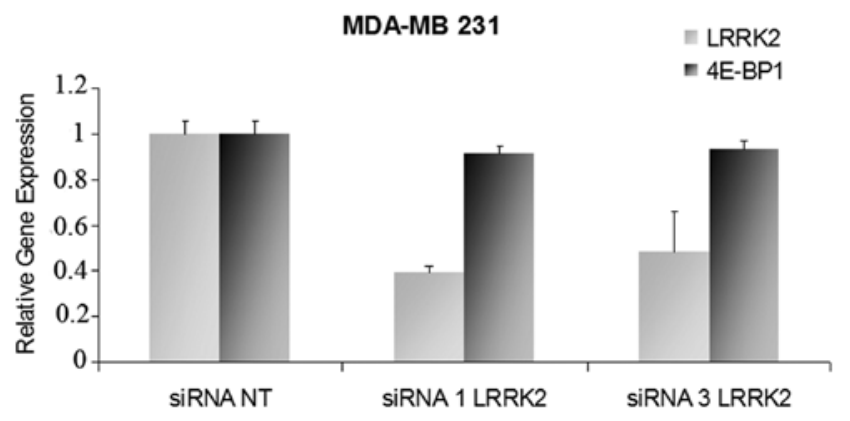

Figure 4. Analysis of 4E-BP1 status by quantitative RT-PCR after inhibition of LRRK2. Levels of 4E-BP1 mRNA were not affected after inhibition of LRRK2 in MDA-MB 231 cell line.

level and stability of the protein. It is likely that mTOR is the main kinase that phosphorylates 4E-BP1 (15) but other, still unidentified, kinases might also be implicated in this event (16-18). In human carcinomas, p-4E-BP1 is overexpressed in a high percentage of tumors regardless of mTOR activation. The novel screening approach used in the present study represented a comprehensive search for new kinases that could be implicated in 4E-BP1 phosphorylation or 4E-BP1 stability. Our findings provide evidence that 4E-BP1 phosphorylation and stability can be regulated by multiple kinases from various cell signaling pathways. Although none of kinases identified were comparable to rapamycin treatment or mTOR inhibition in the ability to specifically affect 4E-BP1 phosphorylation, several kinases did have a significant action in this regard. Moreover, other kinases were found to affect 4E-BP1 stability, with significant decreases in total 4E-BP1 following their inhibition.

Some of the kinases identified here have been found to affect 4E-BP1 phosphorylation in previous studies; these include cyclin-dependent kinase 1 (CDK1) $(16,17)$ and SRC (30). Other previously unreported kinases also seem to have an effect on 4E-BP1 phosphorylation (PDK1, PRKCB1, PAK2, p38 $\beta$, PRKCA and CaMKKB) and interestingly, some of them are implicated in apoptosis. PRKCA and PRKCB1 (also known as PKC $\alpha$ and PKC $\beta$ ) seem to regulate

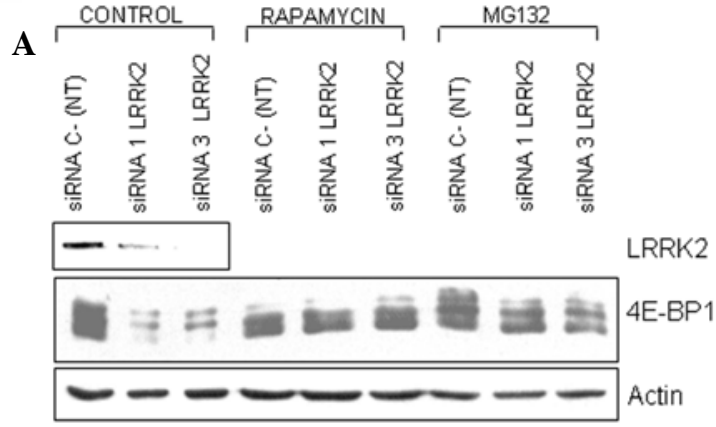

B

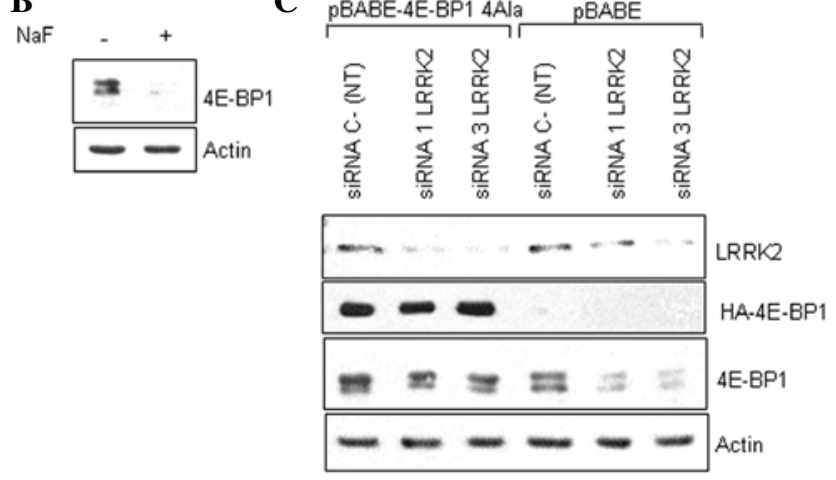

Figure 5. Mode of action of LRRK2. (A), MDA-MB-231 cell line with transiently inhibited LRRK2 was treated with MG132, a proteasome inhibitor, and with rapamycin, an mTOR inhibitor. Both treatments restored total 4E-BP1 protein levels. (B), MDA-MB-231 treated with NaF, a phosphatase inhibitor. The treatment caused total 4E-BP1 down-regulation. (C), MDA-MB-231 cells stably overexpressing a mutant 4E-BP1 [pBABE-4E-BP1 (4ala)]. After LRRK2 inhibition total 4E-BP1 was not down-regulated. Ectopic expression of mutant 4E-BP1 [pBABE-4E-BP1 (4ala)] was confirmed by HA immunoblotting.

multiple pathways, including the MAPK pathway (ERK1/2, JNK and p38). It has been reported that MAPK14/p38 $\alpha$ can phosphorylate 4E-BP1 in HEK293T (31).

With regard to 4E-BP1 stability, a drastic effect was observed with RAF-1 and LRRK2 in MDA-MB-231 cells. We decided to analyze the relation between LRRK2 and 4E-BP1 in more detail because there was a clear decrease in 
4E-BP1 protein levels, but not 4E-BP2 protein. To validate the results obtained with LRRK2 in the screening, we extended our studies to other human cell lines. There was evidence that LRRK2 affected 4E-BP1 stability in 3 other cell lines tested. Moreover, TIG3 cells in which LRRK2 was knocked down proliferated faster than control cells. These findings suggest that an absence of LRRK2 expression and subsequent 4E-BP1 degradation could be relevant in the control of cell proliferation in normal cells. It is known that low levels of 4E-BP1 increase free eIF4E levels for interaction with eIF4G and assembly into the eIF4F complex, resulting in enhanced translation.

Interestingly, down-regulation of 4E-BP1 protein and mRNA has been described when the p38/mitogen-activated protein kinase pathway is activated and in cells treated with PMA. In that model, 4E-BP1 down-regulation was proposed to be mediated by activation of Egr-1 expression through ERK and p38 activation. This effect seems to be specific for 4E-BP1 because levels of 4E-BP2 and eIF4E did not change (32). Similarly our results showed that, after down-regulation of LRRK2, the total amount of 4E-BP2 was not affected, but importantly, after LRRK2 knockdown there was no decrease in 4E-BP1 mRNA. This could indicate that 4E-BP1 regulation through LRRK2 is very specific, occurring only at the protein level, even though other authors have reported that $4 \mathrm{E}-\mathrm{BP} 1$ and 4E-BP2 are regulated by similar pathways $(25,33)$.

A recent study showed that human LRRK2 phosphorylates 4E-BP1 primarily at the Thr $37 / 46$ sites (22). Similarly, Tain et al (34) found that loss of the Drosophila LRRK2 homolog caused 4E-BP1 dephosphorylation. These data further support a link between LRRK2 and 4E-BP1 activity. Nevertheless, the kinase effect of LRRK2 on 4E-BP1 was not observed in other cell lines, such as HEK 293T (31).

We only studied human cells, and lack of LRRK2 protein also confers a proliferative advantage in normal cells, associated with a significant decrease of $4 \mathrm{E}-\mathrm{BP} 1$ protein levels. In our models, we ruled out a direct or indirect transcriptional effect of LRRK2 on 4E-BP1 promoter because the mRNA levels of 4E-BP1 did not change. Our data suggest a model in which 4E-BP1 phosphorylation regulates degradation of this protein. As it is shown in Fig. 5, 4E-BP1 phosphorylation may promote degradation of a fraction of $4 \mathrm{E}-\mathrm{BP} 1$, mediated by the proteasome; this agrees with previous studies in which a role for the proteasome is proposed in 4E-BP1 degradation $(27,29)$. Furthermore, our data suggest that degradation is sensitive to the phosphorylation status of 4E-BP1. The ability of rapamycin to inhibit 4E-BP1 loss and the fact that cells expressing a 4E-BP1 mutant, unable to phosphorylate, did not show the protein decrease after LRRK2 knockdown supports the crucial role of 4E-BP1 phosphorylation in its degradation. Hence, under specific circumstances, stimuli that promote 4E-BP1 dephosphorylation could increase the stability of this protein, and stimuli that promote 4E-BP1 phosphorylation, such as treatment with phosphatase inhibitors, could enhance its degradation.

In summary, a lack of LRRK2 in MDA-MB-231 cells was associated with clearly decreased 4E-BP1 protein (and not with mRNA down-regulation) and increased proliferation in normal cells. The fact that rapamycin treatment can inhibit down-regulation of 4E-BP1 protein offers new insights into the regulation of total and phosphorylated 4E-BP1. It remains to be determined whether the effect of LRRK2 inhibition on
4E-BP1 stability is due to inhibition of a phosphatase or to an ability to direct or indirectly enhance the activity of mTOR or another 4E-BP1 kinase. Further studies will be necessary to explain the ultimate mechanism.

\section{Acknowledgements}

Grant support: Fondo de Investigaciones Sanitarias (Ref. 05/0818 and 08/0143), Fundació Marató TV3 (Ref. 052710), Mutua Madrileña (FMMA/2009/02), Redes temáticas de Investigación Cooperativa en Salud (Ref. RD06/0020/0104 and RD06/0020/1020), and Generalitat de Catalunya (Ref. 2005SGR00144). We would like to thank C. Cavallo for English editing and we greatly appreciate the gift of several LRRK2 plasmid constructs from Bingwei Lu.

\section{References}

1. Avdulov S, Li S, Michalek V, et al: Activation of translation complex eIF4F is essential for the genesis and maintenance of the malignant phenotype in human mammary epithelial cells. Cancer Cell 5: 553-563, 2004.

2. Fingar DC, Richardson CJ, Tee AR, Cheatham L, Tsou C and Blenis J: mTOR controls cell cycle progression through its cell growth effectors S6K1 and 4E-BP1/eukaryotic translation initiation factor 4E. Mol Cell Biol 24: 200-216, 2004.

3. Li S, Sonenberg N, Gingras AC, et al: Translational control of cell fate: availability of phosphorylation sites on translational repressor 4E-BP1 governs its proapoptotic potency. Mol Cell Biol 22: 2853-2861, 2002.

4. Tee AR and Proud CG: Caspase cleavage of initiation factor 4E-binding protein 1 yields a dominant inhibitor of cap-dependent translation and reveals a novel regulatory motif. Mol Cell Biol 22: 1674-1683, 2002.

5. Armengol G, Rojo F, Castellvi J, et al: 4E-binding protein 1: a key molecular 'funnel factor' in human cancer with clinical implications. Cancer Res 67: 7551-7555, 2007.

6. Rojo F, Najera L, Lirola J, et al: 4E-binding protein 1, a cell signaling hallmark in breast cancer that correlates with pathologic grade and prognosis. Clin Cancer Res 13: 81-89, 2007.

7. Castellvi J, Garcia A, Rojo F, et al: Phosphorylated 4E binding protein 1: a hallmark of cell signaling that correlates with survival in ovarian cancer. Cancer 107: 1801-1811, 2006.

8. Castellvi J, Garcia A, Ruiz-Marcellan C, et al: Cell signaling in endometrial carcinoma: phosphorylated 4E-binding protein-1 expression in endometrial cancer correlates with aggressive tumors and prognosis. Hum Pathol 40: 1418-1426, 2009.

9. Graff JR, Konicek BW, Lynch RL, et al: eIF4E activation is commonly elevated in advanced human prostate cancers and significantly related to reduced patient survival. Cancer Res 69: 3866-3873, 2009.

10. O'Reilly KE, Warycha M, Davies MA, et al: Phosphorylated 4E-BP1 is associated with poor survival in melanoma. Clin Cancer Res 15: 2872-2878, 2009.

11. Zhou X, Tan M, Stone Hawthorne V, et al: Activation of the $\mathrm{Akt} / \mathrm{mammalian}$ target of rapamycin/4E-BP1 pathway by ErbB2 overexpression predicts tumor progression in breast cancers. Clin Cancer Res 10: 6779-6788, 2004.

12. Kremer CL, Klein RR, Mendelson J, et al: Expression of mTOR signaling pathway markers in prostate cancer progression. Prostate 66: 1203-1212, 2006.

13. Heesom KJ, Avison MB, Diggle TA and Denton RM: Insulinstimulated kinase from rat fat cells that phosphorylates initiation factor 4E-binding protein 1 on the rapamycin-insensitive site (serine-111). Biochem J 336 (Pt 1): 39-48, 1998.

14. Proud CG: mTOR-mediated regulation of translation factors by amino acids. Biochem Biophys Res Commun 313: 429-436, 2004.

15. Petroulakis E, Mamane Y, Le Bacquer O, Shahbazian D and Sonenberg N: mTOR signaling: implications for cancer and anticancer therapy. Br J Cancer 94: 195-199, 2006.

16. Heesom KJ, Gampel A, Mellor H and Denton RM: Cell cycledependent phosphorylation of the translational repressor eIF-4E binding protein-1 (4E-BP1). Curr Biol 11: 1374-1379, 2001. 
17. Greenberg VL and Zimmer SG: Paclitaxel induces the phosphorylation of the eukaryotic translation initiation factor 4E-binding protein 1 through a Cdk1-dependent mechanism. Oncogene 24: 4851-4860, 2005.

18. Yang DQ and Kastan MB: Participation of ATM in insulin signalling through phosphorylation of eIF-4E-binding protein 1. Nat Cell Biol 2: 893-898, 2000.

19. Gingras AC, Kennedy SG, O'Leary MA, Sonenberg N and Hay N: 4E-BP1, a repressor of mRNA translation, is phosphorylated and inactivated by the $\operatorname{Akt}(\mathrm{PKB})$ signaling pathway. Genes Dev 12: 502-513, 1998

20. Kohn AD, Barthel A, Kovacina KS, et al: Construction and characterization of a conditionally active version of the serine/ threonine kinase Akt. J Biol Chem 273: 11937-11943, 1998.

21. Herbert TP, Tee AR and Proud CG: The extracellular signalregulated kinase pathway regulates the phosphorylation of 4E-BP1 at multiple sites. J Biol Chem 277: 11591-11596, 2002.

22. Imai Y, Gehrke S, Wang HQ, et al: Phosphorylation of 4E-BP by LRRK2 affects the maintenance of dopaminergic neurons in Drosophila. EMBO J 27: 2432-2443, 2008.

23. Gingras AC, Gygi SP, Raught B, et al: Regulation of 4E-BP1 phosphorylation: a novel two-step mechanism. Genes Dev 13: 1422-1437, 1999.

24. Gingras AC, Raught B, Gygi SP, et al: Hierarchical phosphorylation of the translation inhibitor 4E-BP1. Genes Dev 15 2852-2864, 2001.

25. Wang X, Beugnet A, Murakami M, Yamanaka S and Proud CG: Distinct signaling events downstream of mTOR cooperate to mediate the effects of amino acids and insulin on initiation factor 4E-binding proteins. Mol Cell Biol 25: 2558-2572, 2005.
26. Tilleray V, Constantinou $\mathrm{C}$ and Clemens MJ: Regulation of protein synthesis by inducible wild-type p53 in human lung carcinoma cells. FEBS Lett 580: 1766-1770, 2006.

27. Walsh D and Mohr I: Phosphorylation of eIF4E by Mnk-1 enhances HSV-1 translation and replication in quiescent cells. Genes Dev 18: 660-672, 2004.

28. Walsh D, Perez C, Notary J and Mohr I: Regulation of the translation initiation factor eIF4F by multiple mechanisms in human cytomegalovirus-infected cells. J Virol 79: 8057-8064, 2005.

29. Elia A, Constantinou C and Clemens MJ: Effects of protein phosphorylation on ubiquitination and stability of the translational inhibitor protein 4E-BP1. Oncogene 27: 811-822, 2008.

30. Karni R, Gus Y, Dor Y, Meyuhas O and Levitzki A: Active Src elevates the expression of beta-catenin by enhancement of capdependent translation. Mol Cell Biol 25: 5031-5039, 2005.

31. Kumar A, Greggio E, Beilina A, et al: The Parkinson's disease associated LRRK2 exhibits weaker in vitro phosphorylation of 4E-BP compared to autophosphorylation. PLoS One 5: e8730, 2010.

32. Rolli-Derkinderen M, Machavoine F, Baraban JM, Grolleau A, Beretta L and Dy M: ERK and p38 inhibit the expression of 4E-BP1 repressor of translation through induction of Egr-1. J Biol Chem 278: 18859-18867, 2003.

33. Lin TA and Lawrence JC Jr: Control of the translational regulators PHAS-I and PHAS-II by insulin and cAMP in 3T3-L1 adipocytes. J Biol Chem 271: 30199-30204, 1996.

34. Tain LS, Mortiboys H, Tao RN, Ziviani E, Bandmann O and Whitworth AJ: Rapamycin activation of 4E-BP prevents parkinsonian dopaminergic neuron loss. Nat Neurosci 12: 1129-1135, 2009. 